\title{
Medios de contraste intravascular en tomografía computada y resonancia magnética: lo que el clínico necesita saber
}

\author{
Intravascular contrast media in computed tomography and magnetic resonance imaging: \\ what the clinician needs to know
}

Francisco Garrido ${ }^{1}$, Sofía Rivera ${ }^{2}$, José Patricio Pesenti ${ }^{2}$, Carlos Riquelme ${ }^{1}$, Álvaro Huete

\section{Resumen}

Introducción: los medios de contraste en radiología se utilizan para mejorar la visibilidad de los tejidos normales y patológicos, lo que permite distinguirlos entre sí mediante la modificación de las características de imagen de los tejidos. Permite no solo una mejor evaluación morfológica de las lesiones, sino también una evaluación cinética funcional y de contraste. Objetivos: presentar una revisión actualizada sobre los medios de contraste intravascular en radiología, con énfasis en los conceptos que el médico clínico no radiólogo debe tener presente al momento de su indicación. Métodos: se realizó una revisión bibliográfica de literatura radiológica relevante sobre medios de contraste intravascular: clasificación, indicaciones, contraindicaciones y precauciones que debe adoptar el médico clínico. Resultados: aunque los medios de contraste en radiología tienen un excelente perfil de seguridad, su uso no está exento de riesgos y debe basarse en criterios apropiados después de una evaluación clínica exhaustiva, ponderando riesgos y beneficios para cada paciente individual. Conclusión: la comunicación entre médicos tratantes, nefrólogos y radiólogos es fundamental para evaluar casos clínicos complejos o que requieran consideraciones especiales al momento de indicar la administración de un medio de contraste intravascular.

Palabras clave: medios de contraste; medios de contraste yodado; gadolinio; quelatos de gadolinio; daño renal agudo inducido por contraste; fibrosis sistémica nefrogénica.

\begin{abstract}
Introduction: Imaging contrast media are used to enhance the visibility of normal and pathologic tissues, allowing distinction of one another by modifying tissue imaging characteristics. This allows both enhanced morphologic assessment of lesions and also contrast kinetics evaluation. Objectives: To present an updated review on intravascular imaging contrast media. Emphasis will be put on useful concepts that general physicians must take into account when indicating contrast-enhanced imaging studies.

Methodology: A bibliographic review of relevant imaging literature on intravascular contrast media was performed: classification, appropriate indications, contraindications, and tips for proper use by the general physician. Results: Although imaging contrast media have an excellent safety profile, its use is not risk-free and must be based on appropriateness criteria applied after a thorough clinical evaluation, balancing the risks and benefits for each patient. In particular, intravascular contrast media, such as those based on iodine for computed tomography and gadolinium chelates for magnetic resonance imaging, must be used with caution. Conclusion: Effective communication between radiologists and referring physicians is key in the evaluation of complex cases that require protocol adjustments when considering the use of intravascular contrast media for imaging studies.
\end{abstract}

Keywords: Contrast media; iodinated contrast media; gadolinium; gadolinium chelates; contrast-induced acute kidney injury; nephrogenic systemic fibrosis.

Fecha de envío: 25 de octubre de 2019 - Fecha de aceptación: 12 de marzo de 2020

(1) Departamento de Radiología y Diagnóstico por Imágenes, Escuela de Medicina, Pontificia Universidad Católica de Chile, Santiago, Chile.

(2) Escuela de Medicina, Pontificia Universidad Católica de Chile, Santiago, Chile.

Autor de correspondencia: criquelmep@uc.cl 


\section{Introducción}

Actualmente, el uso de los estudios radiológicos se ha convertido en uno de los pilares fundamentales para lograr un diagnóstico certero y rápido frente a problemas de salud de la población, sobre todo en los servicios de urgencia. En este sentido, un estudio realizado en Estados Unidos el año 2008 reportaba que entre 1997 - 2006 existió un incremento en la realización de estudios imagenológicos, sobretodo en tomografía computada (TC) y resonancia magnética (RM), los cuales se duplicaron y triplicaron en el período de estudio respectivamente (Smith-Bindman et al., 2008). Otro estudio del año 2009 publicado en la revista The New England Journal of Medicine describía una realidad similar, con un incremento de un $70 \%$ en la realización de exámenes de imagen entre los años 2000 - 2007 (Iglehart, 2009).

La realidad descrita anteriormente no difiere de lo que ha sucedido en nuestro país. Durante la última década ha existido un progresivo aumento en el número de equipos de imagenología compleja (TC y RM), tanto en el sistema público como privado, lo que ha permitido mejorar el acceso a los métodos diagnósticos por imagen. De acuerdo a las estadísticas de la OECD (Organisation for Economic Co-operation and Development), en Chile el año 2017 existían 446 tomógrafos computados y 226 resonadores magnéticos y el número de estudios de imágenes complejas realizadas entre los años 2007 y 2017 ha aumentado progresivamente, desde 50 a 102 estudios de TC por cada 1000 habitantes y de 5 a 25 estudios de RM por cada 1000 habitantes (OECD Health Statistics, 2019). En nuestra experiencia local, en la Red de Salud UC Christus también ha existido un incremento en la realización de estudios imagenológicos, como señala la figura 1.

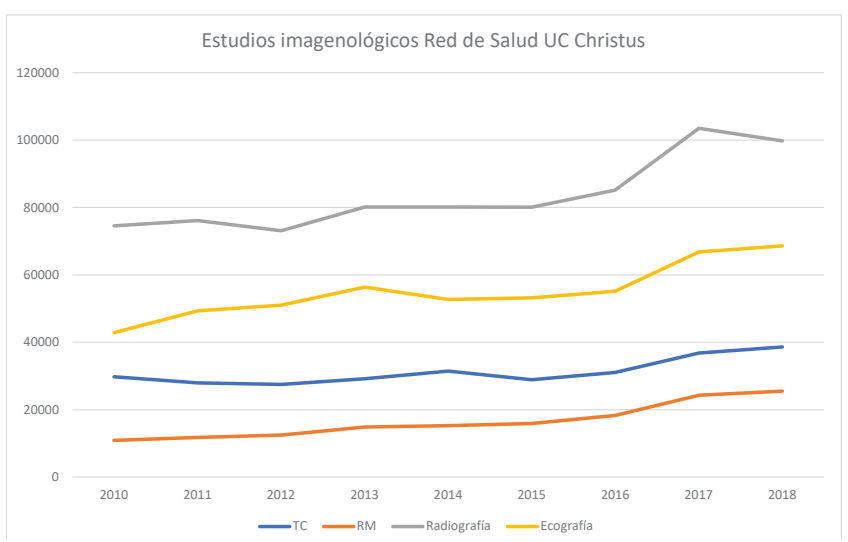

Figura 1. Estudios imagenológicos realizados en la Red de Salud UC Christus (2010 -2018). El gráfico demuestra un aumento en el número de exámenes realizados en todas las modalidades, incluyendo la imagenología completa (TC y RM)
Este incremento en el número de estudios va acompañado de una mayor exposición de la población a los medios de contraste intravasculares utilizados en TC y RM. Es por esto que el personal clínico que solicita estos estudios debe conocer sus características, sus posibles efectos adversos y las condiciones de salud que contraindican su uso.

Los medios de contraste radiológicos son sustancias cuyo único efecto deseado es provocar un cambio en las propiedades de determinados tejidos, cavidades o lesiones para facilitar el diagnóstico mediante distintas modalidades de imagen: modificando la atenuación de los rayos $X$, la intensidad de señal en resonancia magnética ( $R M)$ o la densidad en tomografía computada (TC). La finalidad de los medios de contraste no solo es el estudio morfológico de las estructuras, sino que también aportan información funcional y permiten estudiar el comportamiento vascular de diferentes lesiones en los estudios dinámicos (Méndez et al., 2010).

Al igual que cualquier fármaco, los medios de contraste no son siempre inocuos para el paciente, por lo que su indicación y uso debe ser racional y justificada según la pregunta clínica.

Los medios de contraste yodados son fármacos con un alto perfil de seguridad, con bajo nivel de reacciones adversas. Históricamente se reportaba hasta un 15\% de reacciones adversas al administrar medios de contraste hiperosmolares, sin embargo, actualmente su uso ha sido abandonado. El uso de contrastes yodados isoosmolares y de baja osmolaridad han disminuido el número de reacciones adversas reportadas, las que alcanzan hasta un 0,7\%. Las reacciones adversas severas no superan el 0,04\% (American College of Radiology Committee on Drugs and Contrast Media, 2018). En particular en nuestra institución, en los últimos 10 años se han realizado 98262 inyecciones de contraste, reportando 404 reacciones adversas asociadas al uso de contraste endovenoso, que equivale a un $0,36 \%$. No hay registro de reacciones severas en nuestra institución en los últimos 10 años. Por otro lado, las reacciones adversas a gadolinio fluctúan entre el 0,07\% y 2,4\%, las que en su mayoría son leves (American College of Radiology Committee on Drugs and Contrast Media, 2018). En nuestra institución tampoco se reportan reacciones adversas severas tras la administración de gadolinio en la última década.

\section{Clasificación}

Los medios de contraste utilizados en imágenes se pueden clasificar desde diferentes puntos de vista: 
1. Según la vía de administración:

a. Intravasculares (intraarterial o endovenoso)

b. Endocavitarios (orales, rectales, vaginales, intraarticulares, intracanaliculares o intratecales).

2. Según la capacidad de absorción de rayos X: Esta clasificación corresponde a aquellos medios de contraste usados en estudios que utilizan radiación:

a. Medios de contraste "positivos", que absorben la radiación como el sulfato de bario (utilizado en estudios del tubo digestivo como el enema baritado o la radiografía de esófago-estómago-duodeno) y los contrastes yodados (habitualmente para uso intravascular en tomografía computada o angiografía convencional)

b. Medios de contraste "negativos" como los gases (aire, oxígeno, $\mathrm{CO} 2$, helio) y el agua u otros medios líquidos utilizados para la evaluación de asas intestinales en TC (enterografía o enteroclisis) (Rodríguez et al., 2008).

En el caso de RM, los contrastes también pueden denominarse positivos o negativos dependiendo de la secuencia analizada. Por ejemplo, el agua será un contraste positivo en secuencias ponderadas en $\mathrm{T} 2$ y negativo en secuencias ponderadas en T1. El uso de contrate endovenoso basado en Gadolinio será positivo en secuencias ponderadas en T1, resaltando los órganos en el segmento corporal a estudiar (Ram et al., 2016).

\section{Según su composición química:}

a. Medios de contraste yodados: son aquellos basados en el átomo de iodo utilizados en tomografía computada y estudios angiograficos convencionales (por ej. angiografia cerebral, coronariografía, angiografía renal, etc). Su molécula fundamental es el triyodinato de benceno (Figura 2), que corresponde a un anillo de benceno con tres átomos de yodo y tres radicales que le otorgan la solubilidad en agua (Prieto-Rayo et al., 2014). Pueden estar compuestos por moléculas únicas, es decir un anillo de benceno, denominados monoméricos, o bien por dos anillos benzoicos unidos por un enlace covalente que se mantiene estable en solución, dando origen a los medios de contraste diméricos. Las ventajas de poseer una molécula dimérica es la mayor capacidad de absorción de rayos $\mathrm{X}$ a un mismo volumen de solución al comparar con los monoméricos y la menor generación de iones en solución, con menor actividad osmótica (Prieto-Rayo et al., 2014). b. Medios de contraste basados en Gadolinio: los estudios de resonancia magnética utilizan el gadolinio como elemento fundamental, que corresponde a un metal de alto peso molecular que puede alterar el campo magnético y modificar la señal que emiten los órganos al ser administrado (Thomsen et al., 2018).

4. Según su sitio de acción: según su sitio de acción respecto a la membrana celular, los medios de contraste intravascular pueden ser extracelulares o intracelulares. La mayoría de los contrastes endovasculares son extracelulares, es decir, no atraviesan la membrana celular, por lo que son eliminados por los diferentes órganos sin ser metabolizados, siendo excretados en casi un $100 \%$ por filtración glomerular (Rodríguez et al., 2008). Actualmente existen medios de contraste intracelulares denominados contrastes específicos. El más utilizado en imágenes diagnósticas son los contrastes hepatoespecíficos o hepatobiliares para resonancia magnética (basados principalmente en gadolinio) que son metabolizados por el hepatocito y excretados por la vía biliar. Existen medios de contraste hepatoespecíficos basados en manganeso y en partículas de óxido ferroso, pero ya no son comercializados (Choi et al., 2019).

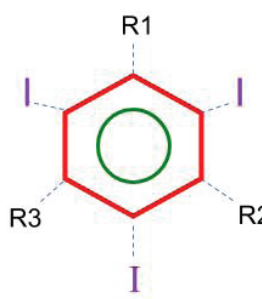

Monomérico

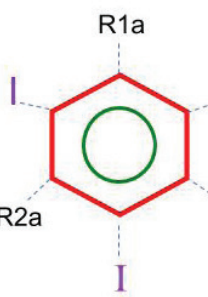

Dimérico

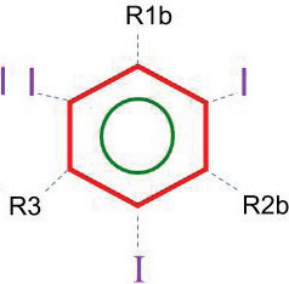

I
Figura 2: Estructura química de los medios de contraste yodados. (Adaptado de Prieto-Rayo et al., 2014)

Las moléculas diméricas, formadas por dos anillos de benceno, a diferencia de los monómeros, atenúan los rayos $\mathrm{X}$ en longitudes mayores y reducen los efectos tóxicos del yoduro libre.

\section{Medios de contraste yodados}

Es uno de los agentes de contraste más ampliamente usado, el cual se utilizada para rayos X (angiografia convencional) y TC. Se inyecta principalmente por vía endovenosa y tiene como efecto aumentar la densidad de la sangre y los tejidos en las imágenes, mejorado la visualización de estos.

\section{Propiedades físico-químicas}

Los medios de contraste intravasculares basados en yodo pueden ser clasificados también segú sus propiedades físico-químicas en una solución (Prieto-Rayo et al, 2014): 
a. Ionicidad: capacidad de disociación de la molécula en una solución, con formación de partículas con carga positiva y negativa, existiendo contrastes iónicos (poseen radicales carboxilos) y no iónicos (poseen radicales hidroxilos).

b. Osmolalidad: concentración de partículas osmóticamente activas en un kilo de agua (Osm/Kg)

c. Osmolaridad: Concentración de partículas en una solución $(\mathrm{Osm} / \mathrm{L})$, pudiendo existir medios de contraste de alta, baja o igual osmolaridad al comparar con la osmolaridad del suero de la sangre humana:

- Alta osmolaridad o hiperosmolares: 1200 a 2400 mOsm/ kg de agua

- Baja osmolaridad: 600 a $800 \mathrm{mOsm} / \mathrm{kg}$ de agua

- Isoosmolares: aproximadamente $300 \mathrm{mOsm} / \mathrm{kg}$ de agua.

La actividad osmótica de los contraste intravasculares determinará la mayoría de sus efectos fisiológicos, siendo mayores en los contraste hiperosmolares, en menor grado en los hipoosmolares y casi ningún efecto en los isoosmolares (American College of Radiology Committee on Drugs and Contrast Media, 2018).

\section{Contraindicaciones al uso de medio de contraste yodado}

Existen contraindicaciones para el uso de medios de contraste yodado endovenoso, las cuales se pueden clasificar en absolutas y relativas. La única contraindicación absoluta para el uso de medios de contraste yodado por vía endovascular es la reacción previa severa al contraste yodado endovenoso (alteración cardiovascular que puede comprometer la vida y que se manifiesta por hipotensión, hipoxia, edema laríngeo, broncoespasmo severo, entre otros). En tanto, las contraindicaciones relativas son aquellas condiciones que otorgan un riesgo mayor al de la población general, porque generan un sustrato que podría potenciar una reacción "tipo alérgica" al medio de contraste yodado, o bien la presencia del medio de contraste yodado en la sangre podría gatillar una descompensación de un enfermedad previa (Beckett et al., 2015).

Dentro de las patologías o condiciones de salud que se consideran como contraindicación relativa se encuentran:

- Historia personal de asma, broncoespamo o atopías. Reacción adversa severa o reacción alérgica severa previa a alimentos y/o medicamentos.

- Historia de insuficiencia cardíaca o daño renal.

- Personas mayores de 60 años y menores de 5 años (American College of Radiology Committee on Drugs and Contrast Media, 2018).

\section{Reacciones al medio de contraste yodado: fisiología y"alergias"}

Las reacciones adversas al medio de contraste yodado pueden ser clasificadas en tipo alérgica y en fisiológicas. La frecuencia de ambas es baja y ha disminuido considerablemente por el uso de medios de contraste modernos (no iónico de baja osmolaridad). La mayoría de las reacciones son leves y no requieren tratamiento (Beckett et al., 2015).

Las reacciones fisiológicas son dependientes de la dosis y concentración del medio de contraste y abarcan desde reacciones vasovagales (bradicardia, hipotensión) hasta arritmias cardíacas y edema pulmonar. La ansiedad y el miedo están asociadas a este tipo de reacción dado que determinan un estado fisiológico predisponente, con exacerbación de las reacciones fisiológicas (taquicardia, liberación de histamina, disnea, entre otros) (Beckett et al., 2015).

Las reacciones tipo alérgicas son independientes de la dosis y concentración del medio de contraste. Tienen una incidencia en general de 1,03\% y en reacción grave de 0,014\% (Suh et al., 2019), aumentando 5 a 6 veces su riesgo de presentar una siguiente reacción (Morcos, 2005). Se desconoce exactamente su mecanismo pero se ha asociado a liberación de histamina.

Se han descrito otros factores de riesgo que aumentan la probabilidad de una reacción adversa al medio de contraste: reacción alérgica severa a comida y medicamentos, historia de asma, broncoespasmo y atopía y antecedente de insuficiencia cardiaca o enfermedad renal. Sin embargo, el Colegio Americano de Radiología no recomienda la premedicación ni la contraindicación del uso de medio de contraste en estas condiciones, dado que el aumento del riesgo no es significativo (American College of Radiology Committee on Drugs and Contrast Media, 2018).

La premedicación se recomienda para aquellos pacientes con antecedente de reacción tipo alérgica o desconocida al mismo tipo de medio de contraste, cuando su administración no retrase la toma de decisiones o el tratamiento. En tanto, no es necesario su administración cuando la reacción alérgica no este relacionada al medio de contraste (como el caso de alergia a los mariscos o al yodo tópico), asma controlada, usuarios de betabloqueadores y otras comorbilidades (American College of Radiology Committee on Drugs and Contrast Media, 2018).

\subsection{Efecto de la premedicación}

La premedicación ha sido estudiada con medios de contraste de alta osmolaridad que ya no se utilizan en la práctica clínica. Sin embargo, la premedicación reduce la probabilidad de una reacción alérgica grave en pacientes de riesgo y reduce también la 
severidad del evento adverso. Hay que tener en consideración que la premedicación no garantiza que no ocurran eventos adversos, estimandose que el número necesario de premedicaciones para evitar una reacción grave es de 369 (American College of Radiology Committee on Drugs and Contrast Media, 2018). No obstante, se han descrito en pacientes hospitalizados que el uso de la premedicación retrasa el examen, aumenta la estadía hospitalaria, los costos y existe un mayor riesgo de infecciones asociadas a la atención de salud (Davenport et al., 2017).

Además, la premedicación no previene todas las reacciones al contraste. Las reacciones fisiológicas no son reducidas por la premedicación y, a pesar de su uso, sigue siendo posible la ocurrencia de una reacción alérgica (conocidas como"breaktrough reactions"). Por tanto, la premedicación no puede reemplazar la preparación del equipo de paro cardiorespiratorio en la sala de examen (American College of Radiology Committee on Drugs and Contrast Media, 2018).

\subsection{Esquemas de premedicación}

En pacientes de alto riesgo de reacción alérgica se utiliza una combinación de corticoides e antihistamínicos no selectivos. La duración mínima de la premedicación no está establecida, con estudios desde 4 horas hasta 12 horas. No hay evidencia que esquemas de menos de 2 horas de duración sean útiles.

El esquema recomendado en la literatura internacional es la premedicación vía oral 12 horas previo al examen con tres dosis (12, 7 y 1 hora antes) de prednisona $50 \mathrm{mg}$ y una dosis de difenhidramina 50 mg una hora antes. En caso de urgencia y ante la imposibilidad de administración por vía oral, consiste en una dosis de metilprednisolona $40 \mathrm{mg}$ / hidrocortisona $200 \mathrm{mg}$ endovenosa 4 horas previo al examen y una dosis de difenhidramina $50 \mathrm{mg}$ una hora antes. Dado que en Chile no está disponible la difenhidramina, se puede utilizar la clorfenamina por vía oral o endovenosa (disponible en ampollas de $10 \mathrm{mg}$ ) (American College of Radiology Committee on Drugs and Contrast Media, 2018).

En pacientes con terapia crónica de corticoides, ACR sugiere reducir la prednisona en los esquemas de premedicación según la dosis de corticoides que reciba el paciente (American College of Radiology Committee on Drugs and Contrast Media, 2018).

\section{Medio de contraste yodado y falla renal}

El daño renal agudo (IRA) inducido por contraste se define como una disminución en la función renal que ocurre días después de la administración intravascular de un medio de contraste yodado. Fisiopatológicamente se cree que existe toxicidad directa del medio de contraste sobre las células tubulares epiteliales, Ilevando a pérdida de función, apoptosis y necrosis. Se han descrito también cambios vasomotores mediados por sustancias vasoactivas que producen alteraciones en la médula renal (Mehran et al., 2019). De acuerdo al grupo de trabajo de Kidney Disease Improving Global Outcomes (KDIGO), se define como el incremento de los niveles de creatinina plasmática en un factor de 1,5 o más sobre el nivel basal dentro de los 7 días posteriores al estudio contrastado, aumento de la creatinina plasmática en al menos $0,3 \mathrm{mg} / \mathrm{dL}$ sobre el nivel basal dentro de las primeras 48 horas o la disminución de la diuresis $\leq 0,5 \mathrm{~mL} / \mathrm{kg} /$ hora que persiste por al menos 6 horas tras la administración de contraste (KDIGO, 2012).

El factor de riesgo independiente más importante para IRA inducido por contraste es la enfermedad renal crónica preexistente, especialmente en aquellos con TFG $\leq 30 \mathrm{~mL} / \mathrm{min} / 1,73 \mathrm{~m}^{2}$, el cual se incrementa en pacientes diabéticos (Rudnick et al., 2019). El uso de contrastes yodados de alta osmolalidad, altas dosis de contraste o inyecciones repetidas también se asocian a un riesgo mayor (Mehran et al., 2019). La vía de administración (endovenosa o intraarterial) no reporta mayores diferencias en el riesgo de desarrollar IRA inducido por contraste, sin embargo, aquellos pacientes sometidos a procedimientos angiográficos tienen riesgo mayor al comparar con el grupo de TC contrastada, por el efecto directo del contraste concretado en la vasculatura renal (Mehran et al., 2019; Rudnick et al., 2019).

Si bien se ha establecido que una incidencia de un $5-6 \%$ de IRA inducida por contraste, hoy se reconoce que la incidencia real de la enfermedad ha sido sobreestimada por las limitaciones metodológicas de los estudios (Mehran et al., 2019). Los medios de contraste yodados utilizados actualmente tienen un muy pequeño riesgo de deterioro de la función renal (Katzberg et al., 2010), por lo que la evaluación clínica juiciosa debera determinar el riesgo particular para cada paciente. La ACR sugiere, en base a la evidencia y opinión de expertos, realizar pruebas de función renal previo a un estudio tomográfico contrastado a los siguientes grupos de pacientes: mayores de 60 años, historia de enfermedad renal (diálisis, trasplante renal, monorreno, cirugía renal, cáncer renal), hipertensión arterial que requiere tratamiento farmacológico, diabetes mellitus y usuarios de metformina (American College of Radiology Committee on Drugs and Contrast Media, 2018).

\subsection{Prevención de falla renal}

La hidratación endovenosa con $\mathrm{NaCl}$ 0,9\% o Ringer Lactato como profilaxis previo al uso de medio de contraste en pacientes de alto riesgo de IRA inducida por contraste está basada en recomendaciones de expertos y es incluida en las guías estadounidenses y europeas (American College of Radiology Committee on Drugs and Contrast Media, 2018; Thomsen et al., 2018). Sin embargo, se debe tener precaución en este este grupo de pacientes (que 
incluye hipertensos, diabéticos y mayores de 60 años), por el riesgo inherente de desarrollar complicaciones por la administración de volumen endovenoso.

Los estudios disponibles comparan distintas terapias de hidratación (como $\mathrm{NaCl}$ 0,9\% y bicarbonato de sodio 1,4\%) e incluyen pocos pacientes con TFG $<30 \mathrm{~mL} / \mathrm{min} / 1,73 \mathrm{~m}^{2}$. Por lo anterior, el estudio AMACING (Nijssen et al., 2017) buscó evaluar la efectividad de esta profilaxis para pacientes con un TFG entre 30 y $60 \mathrm{~mL} / \mathrm{min} / 1,73 \mathrm{~m}^{2}$, concluyendo que el no hidratar profilácticamente no es inferior a la hidratación en la prevención de IRA post contraste. Dado su bajo costo y mínimos riesgos, la expansión de volumen pre contraste se considera un estándar mínimo de prevención en pacientes con alto riesgo de desarrollar daño renal agudo posterior a la administración de medio de contraste yodado endovenoso (Davenport et al., 2015). Se recomienda la administración de solución salina endovenosa entre 6 a 12 horas antes y 4 a 12 horas después del estudio contrastado, lo que es practicable en pacientes hospitalizados (American College of Radiology Committee on Drugs and Contrast Media, 2018).

\section{Situaciones especiales}

\subsection{Medio de contraste yodado y metformina}

La principal preocupación en los pacientes en tratamiento con metformina es el riesgo de acidosis láctica. Dado que la metformina es excretada casi completamente por vía renal suele ser incluida en los cuestionarios previo al uso de medio de contraste por la posibilidad de falla renal. Sin embargo, el ACR establece que los pacientes en tratamiento con metformina no tienen mayor riesgo de desarrollar una IRA inducida por contraste que los pacientes que no la utilizan. Por tanto, los clasifica en dos grupos:

Categoría I: Pacientes sin evidencia de IRA y con TFG $\geq 30 \mathrm{~mL} /$ $\mathrm{min} / 1,73 \mathrm{~m}^{2}$ no necesitan descontinuar la metformina previo ni posterior al uso de medio de contraste.

Categoría II: Pacientes con IRA o enfermedad renal crónica etapas IV o V, o aquellos que serán sometidos a angiografía que puede resultar en embolia en las arterias renales, deben suspender la metformina previo al uso de medio de contraste y las 48 horas posteriores. Solo se debe reiniciar una vez que la función renal haya sido reevaluada. (American College of Radiology Committee on Drugs and Contrast Media, 2018).

\subsection{Embarazo y lactancia}

Los medios de contraste yodados son capaces de atravesar la placenta. Sin embargo, la FDA los clasifica en categoría B, es decir, sin riesgo teratogénico demostrado en estudios con animales pero sin estudios en humanos. No se han reportado casos de hipotiroidismo inducido por el medio de contraste. Además, la lactancia es segura dado que la dosis absorbida de medio de contraste por el feto es menor a 0,01\%. Por tanto, el uso de medio de contraste yodado durante el embarazo y la lactancia no está contraindicado si es necesario para el diagnóstico (Tirada et al., 2015; American College of Radiology Committee on Drugs and Contrast Media, 2018). La precaución a considerar es la exposición a radiaciones ionizantes, lo que si esta comprobado que genera efectos teratogénicos dependientes de la dosis y tiempo de exposición.

\subsection{Pacientes pediátricos}

Si bien los principios y precauciones respecto al uso de medio de contraste yodado en niños y adultos son similares, existen consideraciones especiales. Las reacciones fisiológicas en niños pueden producir irritabilidad o que se muevan durante el examen. Las indicaciones de premedicación son las mismas que en adultos, ajustadas a dosis pediátricas. No existen estudios en niños sobre daño renal agudo inducido por contraste, por lo que se utilizan las indicaciones usadas en adultos (American College of Radiology Committee on Drugs and Contrast Media, 2018).

\subsection{Enfermedad tiroidea}

La tirotoxicosis inducida por medio de contraste yodado es rara. Sin embargo, su uso está contraindicado en pacientes con hipertiroidismo activo. Se sugiere que para pacientes que requieran cintigrafía tiroidea o tratamiento con yodo radiactivo no se utilice medio de contraste yodado por al menos 2 meses previos al procedimiento. Los pacientes con enfermedad de Basedow-Graves o con bocio multinodular deberían ser reevaluados por endocrinología posterior al procedimiento. En pacientes eutiroideos no hay cambios en los niveles de hormonas tiroideas con el uso de medio de contraste yodado (van der Molen et al., 2004; American College of Radiology Committee on Drugs and Contrast Media, 2018).

\section{Medios de contraste basados en gadolinio}

El gadolinio es un metal de la serie de lantánidos con propiedades paramagnéticas que altera el magnetismo de los protones del agua, por lo que se utiliza como componente de agentes de contrastes, mejorando la detección y caracterización de lesiones. En su forma libre es altamente tóxico, por lo que debe unirse a un agente quelante orgánico, convirtiéndose en un medio de contraste inerte y no tóxico que se administra por vía endovenosa en estudios de resonancia magnética y angiografía por resonancia magnética. El complejo gadolinio-quelante se elimina rápidamente por excreción renal en individuos con función renal conservada (Varela et al., 2017). 
De acuerdo al agente ligando, los medios de contraste basados en gadolinio (CBGA) se clasifican en lineales o macrocíclicos, siendo éstos últimos más estables porque el gadolinio se encuentra contenido dentro del ligando, a diferencia de los lineales o de cadena abierta donde el ligando no encierra completamente al gadolinio. Dependiendo de su estructura química, se clasifican en iónicos y no iónicos, siendo los iónicos más estables por las interacciones electrostáticas más fuertes que en los CBGA no iónicos. Por lo tanto, aquellos compuestos macrocíclicos iónicos son más estables, con un mejor perfil de seguridad que aquellos lineales no iónicos (Hao et al., 2012; Choi et al., 2019).

Por otro lado, los CBGA de acuerdo a su biodistribución pueden ser extracelulares no específicos, con distribución en los espacios intersticial e intravascular, los cuales son la mayoría de los CBGA y se excretan por vía renal. Un segundo tipo de CBGA son los que se distribuyen en los espacios extra e intracelular de los hepatocitos, también conocidos como agentes hepatoespecíficos, cuyos representantes son el gadobenato de dimeglumina (MultiHance ${ }^{\circledR}$ ) o gadoxetato de disodio (Primovist ${ }^{\circledR}$ ). Una vez que son metabolizados por los hepatocitos funcionantes, serán eliminados tanto por excreción biliar como renal, propiedad que permite caracterizar lesiones de estirpe hepatocitaria como no hepatocitaria, benignas y malignas (Carrasco et al., 2014; Choi et al., 2019).

\section{Contraindicaciones de uso del gadolinio}

Dentro de las contraindicaciones reportadas en la literatura se encuentran:

- $\quad$ Reacción alérgica grave previa con agentes de medios de contraste basado en gadolinio.

- Embarazo o sospecha de ello en mujeres en edad fértil. Los efectos del gadolinio en el feto son desconocidos, por lo que su uso debe ser con precaución, balanceando riesgos y beneficios. La ACR sugiere dejar registro en la ficha clínica que información se desea obtener con la RM que no pueda ser adquirida sin el uso de contraste, la información es necesaria para el manejo clínico de la paciente o el feto y que la opinión del médico tratante es que no es prudente esperar hasta el fin de embarazo para obtener esta información. La lactancia no es una contraindicación de uso de gadolinio, dado el pequeño porcentaje excretado por la leche materna. Sin embargo, algunas guías recomiendan evitar la lactancia hasta 24 horas después del estudio (Puac et al., 2017)

- Injuria/daño renal agudo.
- Enfermedad renal crónica con una tasa de filtración menor a $30 \mathrm{~mL} / \mathrm{min} / 1,73 \mathrm{~m}^{2}$.

\section{Fibrosis sistémica nefrogénica}

La fibrosis nefrogénica sistémica (FNS) es una trastorno fibrosante multisistémico poco frecuente, que afecta a los pacientes con enfermedad renal crónica expuestos a contraste basado en gadolinio (Bernstein et al., 2012). Puede aparecer días a meses después, con un promedio de 2 a 10 semanas, aunque algunos estudios exponen hasta 8 años (Daftari et al., 2014). Clínicamente puede simular un escleromixedema y se caracteriza por un rápido engrosamiento, endurecimiento e hiperpigmentación progresiva de la piel, principalmente en las extremidades y que progresa desde las piernas y los pies hacia proximal. Además, puede afectar otros órganos extracutáneos como el diafragma, corazón, pericardio, pulmón, tiroides, tracto genitourinario, duramadre, esclera, entre otros (Beckett et al., 2015; Varela et al., 2017).

Si bien la fisiopatología no está totalmente clarificada, se postula que en pacientes con disminución de la función renal, en los cuales los tiempos de aclaramiento son más prolongados, el gadolinio permanece más tiempo en el espacio intravascular pudiendo disociarse del agente quelante, asociado a otros factores metabólicos que ocurren en enfermedad renal avanzada. El gadolinio libre se une a aniones disponibles como el fosfato, generando sales insolubles que se depositan en varios tejidos (Varela et al., 2017). El factor de riesgo más importante para desarrollar FSN es la exposición a CBGA, en dosis única o repetidas, especialmente aquellos donde la unión de gadolinio - complejo quelante sea inestable y la alteración de la función renal. Por lo cual el ACR clasifica los tipos de CBGA según su riesgo de desarrollo de FNS, representados en tabla 1 .

Tabla 1: Clasificación de medios de contraste basado en Gadolinio asociados a fibrosis sistémica nefrogénica según ACR.

\begin{tabular}{l|l|l}
\hline $\begin{array}{l}\text { Clase I: Agentes de } \\
\text { alto riesgo (asociados } \\
\text { con el mayor número } \\
\text { de casos) }\end{array}$ & $\begin{array}{l}\text { Clase II: Agentes de bajo } \\
\text { riesgo (asociados a pocos } \\
\text { casos) }\end{array}$ & $\begin{array}{l}\text { Clase III: Agente de } \\
\text { riesgo indetermina- } \\
\text { do (información al } \\
\text { respecto es limitada) }\end{array}$ \\
\hline $\begin{array}{l}\text { Gadodiamida } \\
\begin{array}{l}\text { Gadopentato de } \\
\text { dimeglumina } \\
\text { Gadoversetamida }\end{array}\end{array}$ & $\begin{array}{l}\text { Gadobenato de } \\
\text { dimeglumina } \\
\text { Gadobutral } \\
\text { Ácido Gadotérico }\end{array}$ & $\begin{array}{l}\text { Ácido Gadoxético } \\
\text { disódico }\end{array}$ \\
\hline
\end{tabular}


En Chile se los CBGA registrados en el Instituto de Salud Pública son: Gadoversetamida (OptiMARK $\left.{ }^{\circledR}\right)$, Gadodiamida $\left(\right.$ Omniscan $\left.{ }^{\circledR}\right)$, Gadopentato de dimeglumina (Magnevist ${ }^{\oplus}$ ), Ácido Gadoxético disódico (Primovist ${ }^{\circledR}$ ), Gadobutral (Gadavist ${ }^{\oplus}$ ) y Ácido Gadotérico (Dotarem ${ }^{\circledast}$ ) (Instituto de Salud Pública, 2018). Sin embargo, la FDA no recomienda el uso de Gadopentato de dimeglumina, Gadodiamida ni Gadoversetamida en pacientes con alteración de la función renal.

La FNS afecta predominantemente a pacientes con enfermedad renal crónica en etapa $V$ en terapia dialítica y en pacientes con daño renal agudo (Bridges et al., 2005). En la literatura hay casos reportados en pacientes con enfermedad renal crónica etapa IV, sin embargo, en estos casos la TFG se encontraba más cercana a $15 \mathrm{~mL} / \mathrm{min} / 1,73 \mathrm{~m}^{2}$ (Moreno-Romero et al., 2007). El último factor es la dosis acumulativa de CBGA, ya que FNS ocurre frecuentemente en aquellos pacientes que recibieron altas dosis, sea como administración única o en múltiples dosis repetidas durante años o meses.

Actualmente no existe un tratamiento específico para esta patología. Se han planteado múltiples opciones terapéuticas, sin embargo la restauración de la función renal es lo único que ha demostrado retardar la progresión de enfermedad. (Beckett et al., 2015). Es por ello que la prevención es muy importante en aquellos pacientes con factores de riesgo, donde se deben privilegiar estudios diagnósticos alternativos que no involucren la administración de gadolinio endovenoso, como la RM no contrastada usando secuencias especiales para ver estructuras vasculares o tejidos patológicos, el ultrasonido, TC no contrastada o TC con contraste y hemodiálisis posterior.

\section{Depósito de gadolinio en el sistema nervioso central}

El año 2014 se reportaron los primeros casos de depósito de gadolinio en el sistema nervioso central, con preferencia por el globo pálido y núcleo dentado. Existe una asociación dosis-dependiente entre la administración de gadolinio y el depósito en el tejido neural, sin embargo, es independiente de la función renal. Hasta ahora se desconoce su fisiopatología y significado clínico (Ramalho et al., 2016; Choi et al., 2019). El depósito de gadolinio puede ocurrir en pacientes con función renal conservada y se asocia con mayor frecuencia a moléculas lineales por su menor estabilidad y mayor tasa de disociación en comparación con las moléculas de estructura macrocíclica (Kanda et al., 2015). Gadodiamida presenta una mayor tasa de depósito en el sistema nervioso central, seguido de Gadopentato de dimeglumina y Gadobenato de dimeglumina (Mathur et al., 2019). A pesar de no existir repercusiones clínicas conocidas, regulaciones internacionales como FDA y EMA recomiendan suspender el uso de medios de contraste basados en gadolinio de estructura química lineal.

\section{Conclusiones}

Los medios de contraste intravascular para TC y RM se consideran fármacos seguros, con una baja tasa de reacciones adversas. Sin embargo, esto no excluye al médico clínico de realizar un completo análisis de los riesgos y beneficios para cada paciente en particular. La comunicación entre médicos tratantes, nefrólogos y radiólogos es fundamental para evaluar casos complejos o que requieran consideraciones especiales.

\section{Fuentes de financiamiento}

No existieron fuentes de financiamiento para esta revisión.

\section{Contribuciones y conflictos declarados por los autores}

Los autores declaran no poseer conflictos de interés.

\section{Referencias}

American College of Radiology Committee on Drugs and Contrast Media. (2018). ACR Manual on Contrast Media. Accedida en https://www.acr.org/Clinical-Resources/Contrast-Manual el 6 de noviembre de 2019.

Beckett K, Moriarity A \& Langer J. (2015). Safe use of contrast media: what the radiologist needs to know. Radiographics 35, 1738-1750.

Bernstein EJ, Schmidt-Lauber C \& Kay J. (2012). Nephrogenic systemic fibrosis: A systemic fibrosing disease resulting from gadolinium exposure. BestPractice \& Research Clinical Rheumatology 26, 489-503.

Bridges MD, St Amant BS, Neil RB, Cernigliaro JG, Dwyer JP \& Fitzpatrick PM. (2009). High-dose gadodiamide for catheter angiography and CT in patients with varying degrees of renal insufficiency: Prevalence of subsequent nephrogenic systemic fibrosis and decline in renal function. American Journal of Roentgenology 192,1538-1543.

Carrasco S, Calles C, Marcin J, Fernández C \& Lafuente J. (2014). Contraste basados en gadolinio utilizados en resonancia magnética. Radiología 56(S1), 21-28.

Choi JW \& Moon W. (2019). Gadolinium deposition in the brain: current updates. Korean Journal of Radiology 20, 134-147.

Daftari Besheli L, Aran S, Shaqdan K, Kay J \& Abujude H. (2014). Current status of nephrogenic systemic fibrosis. Clinical Radiology 69, 661-668. 
Davenport M, Cohan R \& Ellis J. (2015). Contrast media controversies in 2015: imaging patients with renal impairment or risk of contrast reaction. American Journal of Roentgenology 204, 1-8.

Davenport M \& Cohan R. (2017). The evidence for and against corticosteroid prophylaxis in at-risk patients. Radiologic Clinics of North America 55, 413-421.

Girardi M, Kay J, Elston DM, LeBoit PE, Abu-Alfa A \& Cowper SE. (2011). Nephrogenic systemic fibrosis: Clinicopathological definition and workup recommendations. Journal of the American Academy of Dermatology 65, 1095-1106.e7.

Hao D, Ai T, Goerner F, Hu X, Runge V \& Tweedle M. (2012). MRI contrast agents: basic chemistry and safety. Journal of Magnetic Resonance Imaging 36, 1060-1071.

Iglehart J. (2009). Health Insurers and Medical-Imaging Policy - A Work in Progress. The New England Journal of Medicine 360,1030-1037.

Instituto de Salud Pública. (2018). Recomendaciones para la utilización de medio de contraste lineales basados en Gadolinio. Accedida en http://www.ispch.cl/sites/default/files/comunicado/2018/05/ Nota\%20informativa.pdf el 9 de noviembre de 2019.

Kanda T, Oba H, Toyoda K, Kitajima K \& Furui S. (2016). Brain gadolinium deposition after administration of gadolinium-based contrast agents. Japanese Journal of Radiology 34, 3-9

Katzberg RW \& Newhouse JH. (2010). Intravenous contrast medium-induced nephrotoxicity: is the medical risk really as great as we have come to believe?. Radiology 256, 21-28.

Kidney Disease Improving Global Outcomes (KDIGO). (2012). Clinical practice guideline for acute kidney injury. Kidney International Supplements 2,1-138.

Mathur M, Jones JT, Weinreb JC. (2019). Gadolinium deposition and nephrogenic systemic fibrosis: a radiologist's primer. Radiographics 40, 190110.

Mazhar SM, Shiehmorteza M, Kohl CA, Middleton MS \& Sirlin CB. (2009). Nephrogenic systemic fibrosis in liver disease: A systematic review. Journal of Magnetic Resonance Imaging 30, 1313-1322.

Mehran R, Dangas G \&Weisbord, S. (2019). Contrast-associated acute kidney injury. The New England Journal of Medicine 380,2146-2155.

Méndez R \& Graña F. (2010). Fármacos en radiología. En Radiología Esencial, ed. Del Cura JL, Pedraza S \& Gayete A, pp. 65-77. Editorial Médica Panamericana.
Morcos SK. (2005). Review article: acute serious and fatal reactions to contrast media-our current understanding. The British Journal of Radiology 78, 686-693.

Moreno-Romero JA, Segura S, Mascaro Jr JM, Cowper SE, Julià M, Poch E, Botey A \& Herrero C. (2007). Nephrogenic systemic fibrosis: a case series suggesting gadolinium as a possible aetiological factor. The British Journal of Dermatology 157, 783-787.

Nijssen EC, Rennenberg RJ, Nelemans PJ, Essers BA, Janssen MM, Vermeeren MA, Ommen VV \& Wildberger JE. (2017). Prophylactic hydration to protect renal function from intravascular iodinated contrast material in patients at high risk of contrast-induced nephropathy (AMACING): a prospective, randomised, phase 3 , controlled, open-label, non-inferiority trial. Lancet 389, 1312-1322.

Organisation for Economic Co-operation and Development. (2019). Health at a Glance 2019: OECD Indicators. Accedida en https://www. oecd.org/health/health-systems/health-at-a-glance-19991312.htm el 15 de diciembre de 2019.

Puac P, Rodríguez A, Vallejo C, Zamora CA, Castillo M. (2017). Safety of contrast material use during pregnancy and lactation. Magnetic Resonance Imaging Clinics of North America 25, 787-797.

Prieto-Rayo JC \& Valera C. (2014). Farmacología clinica y seguridad de los medios de contraste yodados. Revista de Farmacología de Chile 7, 57-69.

Ram R, Sarver D, Pandley T, Guidry C \& Jambhekar K. (2016). Magnetic resonance enterography: a stepwise interpretation approach and role of imaging in management of adult Crohn's disease. Indian Journal of Radiology and Imaging 26, 173-184.

Ramalho J, Semelka RC, Ramalho M, Nunes RH, AlObaidy M \& Castillo M. (2016). Gadolinium-based contrast agent accumulation and toxicity: an update. American Journal of Neuroradiology 37, 1192-1198.

Rodríguez P, Dena E, Basile R, Fuentes M, Fink G \& Marbez E. (2008). Caracterización fisicoquímica y clínica de los medios de contraste intravasculares iodados. Anales de Radiología México 2, 129-140.

Rudnick MR, Leonberg-Yoo AK, Litt HI, Cohen RM, Hilton S \& Reese PP. (2020). The Controversy of contrast-induced nephropathy with intravenous contrast: what is the risk?. American Journal of Kidney Diseases 75,105-113. 


\section{Garrido et al.}

Smith-Bindman R, Miglioretti D \& Larso E. (2008). Rising use of diagnostic medical imaging in a large integrated health system. Health affairs 27, 1491-1502.

Suh YJ, Yoon SH, Hong H, Hahn S, Kang DY, Kang HR, Choi YH \& Lee W. (2019). Acute adverse reactions to nonionic iodinated contrast media: A Meta-Analysis. Investigative Radiology 54, 589-599.

Thomsen H \& European Society of Urogenital Radiology. (2018). ESUR Guidelines on Contrast Agents. Accedida en http://www.esur. org/guidelines/ el 9 de noviembre de 2019.
Tirada N, Dreizin D, Khati N, Akin E, Zeman RK. (2015). Imaging Pregnant and Lactating Patients. Radiographics 35, 1751-1765. Van der Molen A, Thomsen H, Morcos S. (2004). Effect of iodinated contrast media on thyroid function in adults. European Radiology 14, 902-907.

Varela C, Prieto JC \& Sepúlveda P. (2017). Actualización en medios de contraste endovenosos y riñón. En Avances en Diagnóstico por Imágenes: Urorradiología, ed. Varela C, pp. 1-13. Ediciones Journal. 